\title{
RAISING IN BERBER*
}

\author{
Fatima Sadiqi \\ Université Sidi Mohamed Ben Abdallah \\ Fès, Morocco
}

\begin{abstract}
Raising in Berber has been selected for study for two main reasons: firstly, this syntactic operation which has hardly received any attention up to now involves areas which are central to the understanding of the whole syntactic structure of Berber. Secondly, Raising in general is considered by Chomsky and most linguists as being a universal feature of natural languages. However, although Chomsky does not question the existence of Raising to Subject, which he subsumes under the general rule of NP movement, he seems to strongly reject the rule of Raising to Object, claiming that what this rule does can easily be carried out by means of one of his constraints, the "Tensed S Constraint". The goals of the present paper are twofold: on the one hand, it aims to show that although Chomsky considers Raising to Subject as an essentially movement rule, this rule seems to operate in Berber without syntactic movement. On the other hand, the paper aims to show that Chomsky's theoretical suggestions in the domain of Raising to Object are highly questionable, if not utterly wrong. A number of arguments supporting these two views are based on Berber data.
\end{abstract}

\section{Introduction}

The present paper is an exploration of the way Raising operates in Berber. ${ }^{1}$ The analysis is essentially devoted to formulating arguments against

*I am deeply indebted to Russell G. Schuh (University of California, Los Angeles) and to an anonymous reviewer for their extremely enlightening comments on an earlier version of this paper.

${ }^{1}$ Berber is an Afroasiatic language which is essentially a spoken language without written tradition. The variety of Berber which is analysed in this paper is spoken by the Ayt Hssan tribe, situated in southeast Morocco, near 
Chomsky's views on Raising in general. On the one hand, contrary to what Chomsky claims, Raising to Subject is not always a movement rule; it seems to be some sort of agreement copying rule in Berber. On the other hand, and again contrary to what Chomsky claims, Raising to object should be maintained when analysing natural languages. Evidence lending strong support to these views comes from Berber data.

Raising is a syntactic operation which is closely linked to the whole mechanism of clause complementation. The term "Raising" here is understood within the framework of Standard Theory: it is a movement rule which has the function of extracting an NP from the subordinate complement clause (CC) and locating it in a specific syntactic position in the main clause (MC), either the subject or the object position. This is why Raising is widely known to be of two main types:

Raising to subject, in which case the subject of the CC is raised to the subject position of the MC.

Raising to Object, in which case the subject of the $\mathrm{CC}$ is raised to the object position of the MC.

Fum E??am ${ }^{c}$, some fifty miles south of Azilal. Strictly speaking, this variety has characteristics of both Taslhit and Tamazight (two of the three major dialects of Berber, the third being Tarifit) because, geographically, the Ayt Hssan tribe is situated roughly between the two areas in which the major dialect groups are spoken. The author is a native speaker of this variety. Berber has a rigid unmarked surface VSO order and an alternative SVO order:

(i) i-sya hmad tigmmi 'Ahmed bought the house' he bought Ahmed house

(ii) hmad i -sya tigmmi 'Ahmed bought the house' Ahmed he bought house

Note that verbs in Berber are marked for subject agreement. The absence of overt subjects represented by full NP's makes Berber an instance of "pro-drop" languages (1ike Spanish for instance). But unlike Spanish, independent pronouns such as nttat 'she' in sentences like

$$
\text { nttat } t \text {-ffey brra }
$$
'she went out'$$
\text { she she went out }
$$

do not have an argument status when preverbal. These pronouns are some sort of parentheticals or emphatics. Throughout this paper, the flat structure V NP NP will be assumed. 
The question that poses itself here is, "Is there any evidence for the existence of such rules in Berber?" Let us start by investigating Raising to Subject.

\section{Raising to Subject (R to $S$ )}

The following parameters will be used as tests for the existence of $R$ to $S$ in Berber: verb agreement, subcategorisation, and coordination.

2.1. Verb agreement. Consider the following examples:

(1) a. $t$-dher is $t$-qallaq fadma ${ }^{2}$
she seemed that she angry Fadma

b. $t$-dher is fadma $t$-qallaq she seemed that Fadma she angry

c. $t$-dher fadma is $t$-qallaq she seemed Fadma that she angry

d. ${ }^{*} t$-dher fadma is $t$-qallaq fadma she seemed Fadma that she angry Fadma

e. i-dher is $t$-qallaq fadma

f. $i$-dher is fadma $t$-qallaq it seemed that Fadma she angry

g. $i$-dher fadma is $t$-qallaq it seemed Fadma that she angry

h. * $i$-dher fadma is $t$-qallaq fadma it seemed Fadma that she angry Fadma
'Fadma seemed to be angry'

'Fadma seemed to be angry'

'Fadma seemed to be angry'

'it seemed that Fadma was angry'

'it seemed that Fadma was angry'

'it seemed that Fadma was angry'

According to the above examples, dher 'seem' takes both agreement (la-d) and the neutral subject marker $(1 e-h)$. Agreement holds both when the subject NP fadma is in the CC $(1 a-b)$ and when it is in the MC (1c). The same thing can be said about the neutral subject marker as ( $1 \mathrm{e}-\mathrm{f})$ on the one hand and ( $1 \mathrm{~g}$ ) on the other hand show. No matter whether agreement holds or does not hold, the subject lexical NP cannot be given twice (hence the ungrammaticality of (1d) and $(1 \mathrm{~h})$. When the embedded subject appears in the MC, agreement is slightly

2The word is 'that/whether' is established as a comp in Berber. For evidence, I refer the reader to Sadiqi [forthcoming]. 
preferred because the CC subject is nearer to the MC verb.

Semantically, the sentences with verb agreement and their counterparts with the neutral subject marker are of more or less equal acceptability in the sense that both of them assert that the proposition t-qallaq fadma 'Fadma was angry" wich "seems" or "appears" to the speaker of these sentences is true. The semantic intuition is therefore that the sentences in question are synonymous, though not totally interchangeable: the sentences with verb agreement show more that the subject is involved in whatever is expressed by the MC verb. The semantic relationship between these pairs of sentences justifies their syntactic relationship.

Syntactically, if we compare (1a) and (1c) on the one hand, and (1e) and $(1 \mathrm{~g})$ on the other hand, we will notice that the NP fadma is in the embedded $\mathrm{CC}$ in the first members of the pairs and in the MC in the second members of the same pairs. Thus, the complement subject is clearly raised to the MC subject position. However, this raising does not seem to be achieved by movement given the possibility of agreement/non-agreement in the sentences under study. More specifically, the fact that the MC verb in (1a) can agree with the subject of the embedded CC is good evidence that syntactic movement is not needed for this agreement to take place. Further, the fact that svo is an alternative word order in Berber allows us to say that (1c) can be independently accounted for without positing movement. $R$ to $S$ in Berber seems therefore to be some sort of agreement copying rule. ${ }^{3}$

${ }^{3}$ Note that in a pair of sentences like: $t-/^{*} i$-tyaqqan fadma is $t$-zra fadma baba $s$
she it is sure Fadma that she saw Fadma father her

'Fadma is sure to have seen her father'

$t-l^{*} i$-tyaqqan fadma is $t$-zra baba $s$ she it is sure Fadma that she saw father her

'Fadma is sure to have seen her father'

Raising to Subject is not involved because in both instances, the two subject positions can be filled by independently referring NP's: 
Let's consider verb agreement from another angle. In Berber, when two subject NP's are coordinated, the verb agrees with the first of the conjoined $N P^{\prime} s$, except when the order of constituents is SVO, in which case the verb agrees with the conjunction of the two NP's. It might be worth testing these number agreement facts on the order/agreement variants and see in what way(s) these can help us decide whether the rule of $\mathrm{R}$ to $\mathrm{S}$ operates in Berber or not. Consider the following examples:

(2) a. i -dher $/{ }^{*}$ dher $-n$ is $i-d d a /{ }^{*}$ dda $-n$ hmad $d$ fadma
it seemed seemed they that he went went they Ahmed and Fadma 'it seemed that Ahmed and Fadma went'

b. $\mathbf{i}$-dher $/{ }^{*}$ dher $-n$ is hmad $d$ fadma is dda $-n /{ }^{*} \mathbf{i}-d d a$ it seemed seemed they that Ahmed and Fadma that went they he went 'it seemed that Ahmed and Fadma went'

c. $i$-dher /dher $-n$ hmad $d$ fadma is dda $-n \quad /{ }^{*} i-d d a$ it seemed seemed they Ahmed and Fadma that went they he went 'Ahmed and Fadma seemed to have gone'

d. hmad d fadma $i$-dher /dher $-n$ is dda $-n \quad /{ }^{*} ;-d d a$ Ahmed and Fadma it seemed seemed they that went they he went 'Ahmed and Fadma seemed to have gone'

Sentences (2a,b) are straightforward cases. (2a) is the basic structure. In each example, the top verb is inflected for the neutral marker $i$. As to the inflection on the lower verb, it depends on whether the subject precedes or follows the verb. Examples (2c) and (2d) show that agreement and non-agreement on the MC verb are both possible on1y when the conjoined NP's occur in the MC. More specifically, since the subject in (2c) is plural, the lower verb must therefore be plural. The top verb may either be singular, in which case it is "neutrally" inflected, or plural if it is not so inflected. It should also be pointed out that there is a third option, in which the top verb

(iii) $t-/^{*} i$-tyaqqan fadma is $i$-zra hmad baba $s$ she it is sure Fadma that he saw Ahmed father his

'Fadma is sure that Ahmed saw his father' 
is not neutrally inflected. In such a case, the sentence is fine because that verb agrees with the first member of the conjunct. This is confirmed by the following facts: apart from coordination, verb agreement is also useful when a clause containing one of the $\mathrm{R}$ to $\mathrm{S}$ verbs is embedded within a higher clause. In such cases, dher or bayn 'seem' can either agree with that NP or take the neutral subject marker i 'it'. This is true even when the embedded dher or bayn clauses have their own subjects which are not coreferential with the subject NP. Consider the following examples:

(3) $i$-ssen hmad is dher $-n / i$-dher ifergasn qalqa- $n$ he knew Ahmed that seemed they it seemed children angry they

'Ahmed knew that the children seemed to be angry'

or 'Ahmed knew that it seemed that the children were angry'

(4) i-ssen hmad is i-dher qalqa-n ifergasn he knew Ahmed that it seemed angry they children

'Ahmed knew that the children seemed to be angry' or 'Ahmed knew that it seemed that the children were angry'

In other words, there does not have to be an agreeing subject marker in constructions of the type (4) where the sentence contains an NP embedded within another sentence.

Still within the framework of verb agreement, note that there are verbs which apparently resemble dher and bayn 'seem' in occurring with the neutral subject marker i 'it' but which, unlike them, do not allow verb agreement with the embedded subject no matter whether the latter is in the subordinate $\mathrm{CC}$ or in the MC. Among these verbs, we can cite hlu 'be good, nice', shu 'be true, healthy', $x$ Šen 'be bad, ugly', etc. Here are some examples:

(5) a. i $-l^{*} t$-hla is $t$-dda fadma s ssuq
it she good that she went Fadma to market

'it is good that Fadma went to the market'

b. $\quad \mathrm{i}-{ }^{*} \mathrm{t}-\mathrm{hla}$ is fadma $\mathrm{t}-\mathrm{dda} \mathrm{s}$ ssuq it she good that Fadma she went to market 'it is good that Fadma went to the market' 
c. i $-l^{*}$ hla fadma is $t$-dda s ssuq
it good Fadma that she went to market

'it is good that Fadma went to the market'

Data like the above show that what occurs with verbs like dher and bayn 'seem' is $R$ to $S$. Since verb agreement is not allowed with a verb like hlu 'be good, nice' even when the CC subject is in the MC (5c), then in agreeing with the MC verb dher (and bayn for that matter), fadma in sentences like (1c) must be raised to the MC subject position. This also means that verb agreement does not occur with any verb when the subject occurs in a different clause. It is only when it can in principle occur in the MC subject position that the CC subject can agree with its MC verb. The neutral subject marker i does not really help since. it occurs even with non-Raising verbs. However, since the neutral subject marker alone on the one hand, and verb agreement alone on the other hand, can occur in constructions which do not allow $R$ to $S$, the fact that both of them occur in constructions which involve $R$ to $S$ means that they are significant as a tool for recognising $R$ to $S$ in Berber sentences. If the neutral subject marker is replaceable by the appropriate subject agreement marker in CC's where the embedded subject occurs in the MC, then we have a case of $R$ to $S$.

2.2. Subcategorisation. Another supporting argument for the existence of $R$ to $S$ in Berber is that the two verbs dher and bayn 'seem' are not subcategorised for taking a subject in the normal sense of the term. Compare the following instances:
(6) a. i -ffey hmad
'Ahmed went out'
b. $*_{i}$-dher hmad
'Ahmed seemed'
he seemed Ahmed

Note that 1ike 'appear' in English, dher and bayn 'seem' have two meanings: the one that has been used up to now, and the one in which some quick visual appearance is involved. (6b) is ill-formed with the first meaning but all right with the'second one. However, in the present case, the second meaning is irrelevant since it does not involve any clause complementation and thus 
should not be taken to relate to the subcategorisation of the dher and bayn we are considering.

The two verbs dher and bayn usually occur only as main clause verbs followed by a complement clause. Their subcategorisation can be represented as follows:

(7) dher / bayn $\rightarrow$ [ CC 非 ${ }^{4}$

However, if we go back to $(1 \mathrm{c}),(1 \mathrm{~g})$, etc., we shall notice that the above subcategorisation seems, at first sight, to be violated since dher in these instances is followed by an NP. A closer look at the examples in question reveals that this violation is only apparent and can be accounted for. If we postulate a rule of $R$ to $S$ which explains the positioning of the CC subject in the MC subject position and if we assume that subcategorisation takes place in the underlying structure where the CC subject is still with its clause coelements, then no violation of the subcategorisation frame of dher (and bayn) has occurred.

2.3. Coordination. A further argument supporting the existence of the rule of $\mathrm{R}$ to $\mathrm{S}$ in Berber is that the NP which originates as the CC subject can be the subject of two coordinated predicates:

$$
t \text {-uhl fadma aha } t-/ i \text {-dher is } t-i \text {-ay kra }
$$
she is tired Fadma and she it seemed that her it affected something

'Fadma is tired and seems to be $i 11$ '

or 'Fadma is tired and it seems that she is $i 11$ '

On the basis of verb agreement, Fadma in the above example seems to function as both the subject of uhl 'be tired' and also of dher. However, since the subject agreement marker is retained on the lower verb, $\mathrm{R}$ to $\mathrm{S}$ looks like an agreement copying rule, not a movement rule.

\footnotetext{
${ }^{4}$ However, given that sentences like (1a) are possible in Berber, another possible subcategorisation for dher and bayn 'seem' could be:$$
\text { [s } \mathrm{AGR}_{i}\left[\begin{array}{llll}
\mathrm{S}_{\mathrm{S}} & \mathrm{X} & \mathrm{NP}_{i} & ]
\end{array}\right]
$$

but the NP here does not occur in the same clause that dher appears in. 
At the end of this section, which has presented some arguments in favour of the existence of $R$ to $S$ as an agreement copying rule in Berber, let us add that from the semantic point of view, sentences like ( $1 a$ ) and ( $1 b$ ) on the one hand, and sentences like $(1 \mathrm{~g})$ on the other hand are understood in such a way that the MC subject NP and the following clause form jointly one single semantic unit or "clause" in this context. The sentence fadma is t-qallaq is indeed one such unit if we compare it to $t / i$-dher fadma for instance. Therefore, the logical or underlying structure of ( $1 \mathrm{a})$ and (1b) on the one hand, and $(1 \mathrm{~g})$ on the other hand should clearly indicate that it consists of two parts, $[t / i-d h e r]$ and [fadma is $t$-qallaq], and that these two parts are connected in some way. Following from this and supporting the claim that in CC's, when $R$ to $S$ takes place, the sentence from which the subject NP is raised always functions as the logical subject of the sentence, we can represent this relationship in the following terms:

(9)

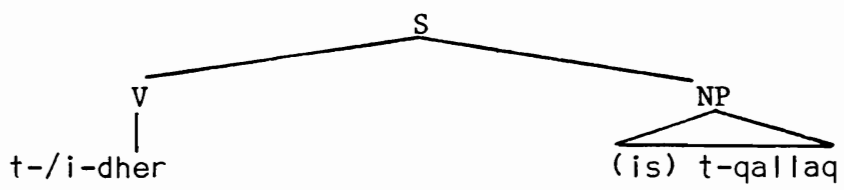

Overall then, there is enough evidence to support the existence of $R$ to $S$ in Berber. This rule obviously participates in subject formation and accounts for a number of syntactic phenomena relating to the subject. The fact that only two verbs trigger $R$ to $S$ and the fact that this rule does not cause any change in the tense, mood, etc. of the sentence in which it operates might suggest that although $R$ to $S$ exists in Berber, the rule is rather restricted. However, this seems to be a general feature of the rule itself. Let's add here that $\mathrm{R}$ to $\mathrm{S}$ operates only in declarative $\mathrm{CC}$ 's since the two verbs which trigger this rule take neither interrogative nor infinitive CC's.

2.4. $\mathrm{R}$ to $\mathrm{S}$ Vs Subject Equi. $\mathrm{R}$ to $\mathrm{S}$ should be sharply distinguished from subject Equi. The latter basically involves the deletion of subject under identity. $R$ to $S$ is different from Equi in the sense that it does not delete the CC subject NP. Note in relation to Equi that this rule might not be relevant 
in Berber since subject agreement markers always appear on the verb, a fact which means that the subject in Berber sentences is never totally lost. A very general problem must be kept in mind here: whereas in English, for example, we know when Equi has applied, in Berber we cannot really distinguish it from the simple anaphoric absence of the full NP (all relevant functions being fulfilled by the subject agreement markers). More specifically, given a Berber sentence like

$$
\begin{aligned}
& \text { i -ssen hmad is ma i -njah } \\
& \text { he knew Ahmed that FUT he succeed }
\end{aligned}
$$

we do not know whether it is subjectless, that is, corresponding to the non-Eng lish *'Ahmed knew to succeed', or whether it more directly corresponds to 'Ahmed knew (that) he would succeed'. This is part of the same problem as whether verbs with just subject agreement markers are subjectless or not. I shall continue to use the term "Equi" bearing in mind that in principle, it does not mean in Berber what it means in English or any other similar language.

3. Raising to Object ( $R$ to 0 )

Consider the following examples:

a. i -ssen muha is i -ara hmad tabrat he knew Muha that he wrote Ahmed letter 'Muha knew that Ahmed wrote the letter'

b. i-ssen muha hmad is i -ara tabrat he knew Muha Ahmed that he wrote letter

'Muha knew that Ahmed wrote the letter'

According to the above examples, the following operation seems to have taken place:

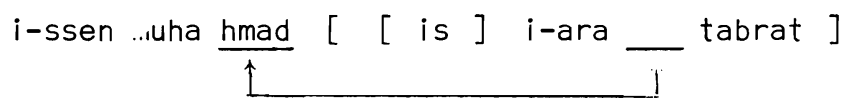

Thus, hmad, which originates as the CC subject in (11a), seems to act as the MC direct object in (11b). To confirm this, we need tests which are capable of showing that the "moved" NP is indeed the MC direct object and that the bracketing occurs before, not after, the comp is. The following tests will be 
used: verb agreement, passivisation, reflexivisation, clefting, and non-recursiveness.

3.1. Verb agreement. As we are investigating $R$ to 0 , the test of verb agreement will be mainly based on the behaviour of the direct object clitic. $t$ 'him/her/it'. Note that tnere is a crucial difference between object (direct and indirect) clitics and subject agreement markers: as their name indicates, subject markers are essentially agreement markers while object clitics are pronominalised forms of full NP's. The latter have full argument status, but there is no evidence that the former do. Further, whereas subject agreement markers are obligatory, object clitics are not:

Direct object clitics in Berber can either replace direct object lexical $\mathrm{NP}^{\prime} \mathrm{s}$ or copy them if the latter are preposed to the preverbal position, i.e. if the sentence is ovs:
(13) a. i -ara muha tabrat
he wrote Muha letter
b. i -ara -t muha he wrote it Muha
c. tabrat i -ara -t muha 'Muha wrote the letter' letter he wrote it Muha
'Muha wrote the letter'
'Muha wrote it'

In either case, as the above examples show, the direct object clitics get attached to the verb. Further, in the first case, the clitic carries specific semantic information whereas in the second case it marks a specific word order in Berber sentences. Note that when the direct object clitic replaces a full lexical NP, it cannot co-occur with it. If, for example, we replace the $\mathrm{NP}$ hmad in (11b) by the direct object clitic, we shall have:

(14) i-ssen-t muha (*hmad) is i -ara tabrat he knew him Muha Ahmed that he wrote letter

'Muha knew him to have written the letter'

Note incidentally that the direct object clitic cannot.co-occur with hmad even if the latter stays in its clause of origin:

(15) i-ssen-t muha is i -ara (*hmad) tabrat he knew him Muha that he wrote Ahmed letter

'Muha knew him to have written the letter' 
Needless to say, the lexical NP hmad cannot occur in both the CC and the MC at the same time.

Since the direct object NP and its corresponding direct object clitic cannot co-occur in the same construction when the latter replaces the former as (14) and (15) show, then some operation across clauses must have taken place. Further, since the direct object clitic does not attach to the embedded verb, then it logically does not belong to the embedded clause, in which case it automatically belongs to the MC.

Another thing to note is that the complement subject in sentences like

(11b) can occur in the very initial position of the construction:

(16) hmad i -ssen-t muha is i -ara tabrat

Ahmed he knew him Muha that he wrote letter

'Muha knew that Ahmed wrote the letter'

In such cases, the presence of the direct object clitic is obligatory because the MC seems to have an OVS order. This is more evidence that the CC subject in sentences like ( $11 \mathrm{~b}$ ) is moved to the MC object position because otherwise it would not be able to participate in making the MC an OVS sentence and would instead do so with the CC. In other words, the NP in question is capable of moving within the $\mathrm{MC}$ and not the $\mathrm{CC}$ and this constitutes evidence that it pertains to the former.

Overall then, so far as verb agreement is concerned, the newly moved NP seems to take on the properties of direct object NP's. Note incidentally that as with $\mathrm{R}$ to $\mathrm{S}$, pairs of sentences like (11a) and (11b) are synonymous.

In order for us to really show that $R$ to 0 exists in Berber, we should be able to demonstrate that some sort of sentential boundary occurs between the newly moved NP and the comp introducing the CC or, in the absence of an overt realisation of the latter, between the MC object and the verb which was its erstwhile mate. To test this, two rules which are known, on independent evidence, to be clause internal in Berber, passivisation and reflexivisation, will be used. If the newly moved NP can participate in these two rules, then it belongs to the $\mathrm{MC}$. 
3.2. Passivisation. Passivisation is a rule which rearranges constituents within clause boundaries. In Berber, passivisation has the role of promoting the object of a sentence to the subject position and then deleting the subject altogether. Consider the following examples:

(17) a. i -ssen muha hmad is i -ara tabrat he knew Muha Ahmed that he wrote letter

'Muha knew that Ahmed wrote the letter'

b. i -tya -ssan hmad is i -ara tabrat he passive known Ahmed that he wrote Ietter

'Ahmed was known to have written the letter'

Given that hmad is moved to the object position in (17a) and after passivisation this same object becomes the subject of the passivised verb in (17b), then hmad seems to belong to the MC since the rule of passive operated within the boundaries of the MC. This point can be argued for more forcefully. In a sentence like

(18) $i$-ssen muha tabrat is $t$ - $i$-ara hmad he knew Muha letter that it he wrote Ahmed

'Muha knew that Ahmed wrote the letter'

the point is that tabrat is a topicalised ${ }^{5}$ object in the CC and therefore Raising, which applies only to subjects in the CC, cannot have applied to it. This is confirmed by the fact that it cannot passivise:

* $t$-tya -ssan tabrat is $t-i$-ara hmad

it passive known letter that it he wrote Ahmed

'the letter was known to have been written by Ahmed'

Note that, interestingly, the situation is different with NP's starting as CC subjects. Consider the following construction in which passivisation takes place within the CC:

(20) i -ssen muha is $t$-tya -ara tabrat he knew Muha that it passive written letter

'Muha knew that the letter was written'

${ }^{5}$ Topicalisation will be dealt with in section 4 . 
(20) can undergo $\mathrm{R}$ to 0 :

(21) i -ssen muha tabrat is t -tya -ara

he knew Muha letter that it passive written

'Muha knew that the letter was written'

and can thus passivise:

t -tya -ssan tabrat is t -tya -ara

it passive known letter that it passive written

'the letter was known to have been written'

Note that in this particular case, the rule of $\mathrm{R}$ to 0 must precede the rule of passive because it supplies the appropriate object which eventually becomes the subject of the passivised verb. Thus, if we compare (22) and (19), we sha11 notice that passivisation is blocked in the latter because the NP tabrat starts as the object of the embedded verb ara in the same construction (the subject being hmad), and being the object of the $\mathrm{CC}$, tabrat cannot be the object of the MC and hence cannot participate in the passivisation of the latter. By contrast, passivisation is not blocked in (22) because the NP tabrat starts as the subject of the CC (cf. 20), then gets raised to the MC object position (cf. 21), and thus legitimately participates in the passivisation of the MC (cf. 22).

Overall then, passivisation constitutes strong evidence that $R$ to 0 exists in Berber. It is possible in CC constructions only when the CC subject is raised to the $M C$ object position or within the boundaries of the subordinate CC. Further, since passivisation is inherently limited to clause internal operations, the fact that it involves the CC subject in a sentence like (22) means that this subject belongs to the $M C$ and that $R$ to $O$ is justified.

3.3. Reflexivisation. Reflexivisation ${ }^{6}$ is a means of marking coreferentiality. Berber marks it only up through one single clause. In other words, re-

\footnotetext{
${ }^{6}$ Berber has two types of reflexives, (1) $s$ (with) + ixf (head) + possessive pronoun and (2) ixf (head) + possessive pronoun. These reflexives inflect for the categories. of person, number, and gender of the noun they
} 
flexives in Berber seem to always occur in the same clause with the referents to which they are anaphors:

(23) a. i-iyal hmad is i-uf akk mdden he thought Ahmed that he was best of all people

'Ahmed thought that he was the best of all people'

b. *i-iyal hmad is ixf ns $i$-uf akk mdden he thought Ahmed that head his it was best of all people * 'Ahmed thought that himself was the best of all people'

c. i-iyal hmad ixf ns is i -uf akk mdden he thought Ahmed head his that it was best of all people

'Ahmed thought himself to be the best of all people'

The reflexive pronoun cannot occur as the subject of the CC, hence the ungrammaticality of (23b). The occurrence of this pronoun in (23c) is significant. In fact, since reflexivisation in Berber applies only to objects (direct or indirect) and since reflexive pronouns in sentences like (23c) act as direct objects of the MC but bear a structural relation to the CC (cf. the subject agreement markers), then they must be former CC subjects which achieved their final locus through the operation of $R$ to 0 . The fact that the process of re-

replace, which makes the latter easily traceable. The following are illustrative examples:

$$
\text { zri- hmad } s \text { ixf ns *ixf ns }
$$
saw I Ahmed with head his head his

$$
\begin{aligned}
& \text { i -jrah hmad ixf ns /*s ixf ns 'Ahmed cut himself' } \\
& \text { he cut Ahmed head his with head his }
\end{aligned}
$$

The first type of reflexives functions as an intensifier of the NP it follows, whatever the function of the latter is. As to the second type, it replaces an NP which is identical to the subject NP but which functions as the object (direct or indirect) of that NP. This is why whereas the first type can be replaced by an adjective like undit 'alone', the second type cannot and can instead be replaced only by an $\overline{\mathrm{NP}}$ of some sort:

(iii) i -dda hmad uhdit/*fadma/*acyyal ad /etc. 'Ahmed went alone' he went Ahmed alone Fadma boy this

(iv) i -jrah hmad fadma/acyyal ad /*uhdit/etc. 'Ahmed wounded Fadma/
he cut Ahmed Fadma boy this alone this boy/etc.' 
flexivisation is carried out within the boundaries of the MC clearly indicates that reflexive pronouns in.such cases belong to the $M C$ and that there is no clausal boundary between the MC subjects and their reflexive pronouns.

3.4. Clefting. Clefting in Berber is a movement rule whose function is to 1ocate a constituent in the focus position which happens to be the initial position in Berber and introduce the cleft marker a immediately after it. ${ }^{7}$ In Berber, constituents cannot be clefted out of subordinate clauses:

(24) a. i -ssen muha is i -ara hmad tabrat he knew Muha that he wrote Ahmed letter

'Muha knew that Ahmed wrote the letter'

b. *tabrat a i -ssen muha is i -ara hmad letter cleft marker he knew Muha that he wrote Ahmed 'it was the letter that Muha knew Ahmed had written'

On the basis of this, an argument supporting the existence of $\mathrm{R}$ to 0 in Berber is that if we move the CC subject NP hmad to the pre-comp position, it can undergo clefting:

(25) a. i -ssen muha hmad is i -ara tabrat he knew Muha Ahmed that he wrote letter

'Muha knew that Ahmed wrote the letter'

b. hmad a i -ssen muha is i -ara tabrat Ahmed cleft marker he knew Muha that he wrote letter 'it was Ahmed that Muha knew had written the letter'

The fact that hmad in (25b), which originates as the CC subject (cf. 25a), can be subject to clefting confirms the assumption that it no longer belongs to the $\mathrm{CC}$ and that it is a constituent of the MC because if it were part of the subordinate clause, it would not cleft (cf. 25b).

3.5. Subcategorisation. Subcategorisation provides further evidence for the existence of $R$ to $O$ in Berber. As in English, for example, some verbs in Berber take $R$ to 0 and others do not. A verb like amn 'believe' can be fol-

${ }^{7}$ the reader is referred to Ennaji and Sadiqi [1986]. 
lowed by either an object NP or a CC:

(26) a. umn $-\gamma$ hmad

believed I Ahmed

'I believed Ahmed'

b. umn $-\gamma$ is $i-n j a h$ hmad $g$ limt ihan

believed $I$ that he succeeded Ahmed in exam

'I believed that Ahmed passed his exam'

This would exclude just inanimate objects appearing without the CC:

(27) *umn $\quad-\gamma$ tigmmi

believed I house

*'I believed the house'

If we take (26b) as the starting sentence, then the following two sentences are the ones which suggest $R$ to 0 :

(28) a. umn $-\gamma$ hmad is i -njah

believed I Ahmed that he succeeded in exam

'I believed Ahmed to have passed his exam'

b. umn $-\gamma-t$ is $i-n j a h$ g limt ihan

believed I him that he succeeded in exam

'I believed him to have passed his exam'

If we assume that the NP hmad in (28a) above and the direct object clitic in ( $28 \mathrm{~b}$ ) are not derived by the movement rule of $\mathrm{R}$ to 0 but by derivation, then we shall have tremendous problems with subcategorising verbs like ija 'think, believe' which do not take animate objects

(29) *iyal - - hmad/flan / kra n urgaz thought I Ahmed someone some of man

*'I thought Ahmed/someone/some man'

but which take a CC

(30) iyal -y is i -njah $g$ limtihan

thought $I$ that he succeeded in exam

'I thought that Ahmed passed his exam' 
and allow the movement of hmad to the MC. Compare (31) with (28) above:

(31) a. iyal $-\gamma$ hmad is i -njah g limt ihan

thought I Ahmed that he succeeded in exam

'I thought Ahmed to have passed his exam'

b. iyal $-\gamma-t$ is i-njah $g$ limtihan

thought $I$ him that he succeeded in exam

'I thought him to have passed his exam'

However, if we assume that subcategorisation applies to verbs in underlying structure and that the moved NP hmad in (31a) or its corresponding direct object clitic in (31b) originate as the CC cuoje. (.). 30), then the subcategorisation of iyal in cases like (31a) aind ( $31 b$ ) is not violated. In other words, since $R$ to $O$ depends on subcategorisation, then movement is involved, and if the moved NP were still in the subordinate clause, then the MC verb should not affect what is moved in the embedded clause. Thus, it is only by positing a rule of $R$ to 0 that the problem of subcategorising verbs like iyal, which do not take animate objects, can be solved.

3.6. Non-recursiveness. A characteristic of the rule of $R$ to 0 is that it is non-recursive in the sense that it is restricted to crossing constituents over only the immediate superordinate clause. This is compatible with the fact that, generally speaking, unlike subject NP's, object NP's are not supposed to move freely up in the structural tree. In what follows, we shall test these properties of the rule of $R$ to 0 on Berber sentences and find out whether the existence of $R$ to $O$ in Berber is or is not confirmed. Consider the following instances:

(32)
a. $\mathrm{i}^{\mathrm{i}-\mathrm{bayn}}$
$\uparrow^{t}$-nna fadma $\uparrow^{i}$-ssen hmad
is $t$-qallaq malika
it seemed she said Fadma he knew Ahmed that she angry Malika
'it seemed that Fadma said that Ahmed knew that Malika was angry'
b. $i$-bayn $t$-nna fadma $i$-ssen hmad malika is $t$-qallaq
it seemed she said Fadma he knew Ahmed Malika that she angry
'it seemed that Fadma said that Ahmed knew Malika to be angry' 
The NP malika, which functinns as the subject of the most embedded sentence in (32), can move only to the next higher sentence. In other words, this NP cannot be separated from its original co-elements unless it moves just one step higher in the structural tree given that its movement to the other clause is blocked (cf. 32a). This is shown in the following configuration:

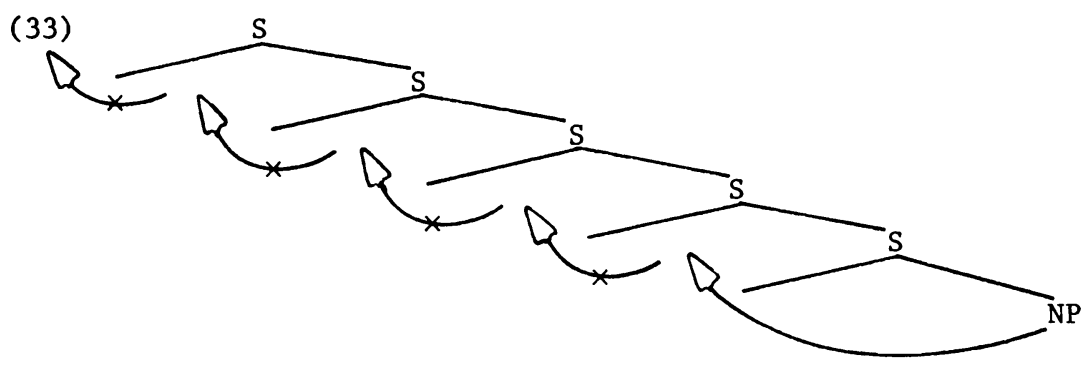

Since the rule of $R$ to 0 in Berber is not recursive, it is obviously bounded. One should bear in mind however that presumably, alternate occurrences of $R$ to 0 and Passive, for example, will allow repeated applications of the rule. Thus, a further argument for the existence of $R$ to 0 in Berber is that this rule is non-recursive.

Overall then, all the arguments given in favour of the existence of the rule of $R$ to $O$ in Berber seem to indicate that the sequence $N$ COMP $V \quad X$ is not organised as a clause in Berber when the embedded subject is raised to the matrix clause, and only the part following the comp is the subordinate clause. In other words, the arguments given in support of the existence of $R$ to 0 in Berber seem to indicate that there is a clause boundary before comp in CC constructions involving $R$ to 0 . The NP raised to the MC object position occurs in all the positions in which the object NP occurs and also feeds into various other rules which can be applied to ordinary objects. The rule thus participates in object formation in Berber.

$R$ to 0 operates in infinitive ${ }^{8} \mathrm{CC}^{\prime} \mathrm{s}$ as well:

${ }^{8}$ The notion of "infinitive" in Berber is an important issue, but for lack of space, I shall limit myself to the following: formally, the obligatory occurrence of the subject agreement markers on verbs makes it difficult to talk 
(34) a. i-ra muha a i -ara hmad tabrat

'Muha wanted Ahmed to write the letter'

b. i-ra muha hmad a i -ara tabrat

he wanted Muha Ahmed to he write letter

'Muha wanted Ahmed to write the letter'

Everything that has been said about the operation of $R$ to 0 in declarative CC's, including the replacement of the moved NP by the direct object clitic $t$ 'him/her/it', applies to infinitive CC's.

$R$ to $O$ in infinitive CC's should be distinguished from another phenomenon which might apparently be confused with it. Consider the following examples:

(35) a. i -ra muha hmad a i -izar atbib

he wanted Muha Ahmed to he see doctor

'Muha wanted Ahmed to see the doctor'

b. i $-z z^{c}$ am muha hmad a i -izar atbib

he urged Muha Ahmed to he see doctor

'Muha urged Ahmed to see the doctor'

Sentence (35a) involves $R$ to 0 (the embedded subject hmad is raised to the MC direct object position). As to (35b), it involves Equi NP deletion (the embedded subject is deleted under coreferentiality with the direct object of the MC).

In spite of the fact that $R$ to 0 does not occur with verbs 1 ike $z z^{C}$ am 'urge', Equi NP deletion can optionally delete the CC subject of verbs like iri 'want' if it happens to be coreferential with the MC subject:

about finite/non-finite clauses in Berber. However, from the grammatical point of view, the difference between the two clauses can be clearly shown on the basis of tense. The verb in Berber can occur in five tenses (past, "used to" form, present progressive, present habitual, and future). Each of these tenses is characterised by a specific form. In addition, the verb in Berber can occur in one of the following forms: / $\mathrm{i} n /$ form (participial form) and $a+V$ (infinitive form). These forms are invariable, they never occur in $V$ positions, and they are semantically incomplete. They are thus non-finite, as opposed to the finite forms. 
Note that $R$ to 0 can operate in a sentence like (36) provided a reflexive pronoun is introduced because in such cases, the MC subject and direct object are identical:
i -ra
hmad ixf ns a i -ddu
'Ahmed wanted himself to go'
he wanted Ahmed head his to he go

$R$ to $O$ and Equi NP deletion do not apply freely to every verb in every context There, thus, has to be some way of specifying for each verb which rule(s) it can undergo and in what $\operatorname{context}(\mathrm{s})$.

3.7. $\mathrm{R}$ to 0 vs. Embedded Object Movement. Apart from the subject NP, the CC in Berber may include an object NP which can move to the pre-comp position, in which case it can easily be taken for a raised NP. This phenomenon occurs in the three types of $\mathrm{CC}$ as the examples below indicate:

(38) a.

$i$-ssen muha tabrat is $t-i$-ara hmad

9

he knew Muha letter that it he wrote Ahmed

'Muha knew that Ahmed wrote the letter'

b. i -ra muha tabrat a $t-i$-ara hmad

he wanted Muha letter to it he write Ahmed

'Muha wanted Ahmed to write the letter'

c. $i$-seqsa muha tabrat is $t$ - $i$-ara hmad

he asked Muha letter whether it he wrote Ahmed

'Muha asked whether Ahmed had written the letter'

${ }^{9} \mathrm{~A}$ note on the positioning of the verb "satellites" in Berber is in order here. The subject agreement markers have fixed positions (the first person subject agreement marker follows the verb stem whereas the third person subject agreement markers precede the verb stem, and the second person subject markers both precede and follow the verb stem). As to the object clitics (direct and indirect), they are movable. They follow the verb stem and the subject agreement markers if the verb is initial in a sentence; otherwise, if the verb is preceded by a pronoun, a comp, etc., the object clitics precede the verb stem and the subject agreement markers. In both cases, the indirect object clitics precede the direct object clitics. 
Our task is thus to investigate the nature of this CC object movement and find out the extent to which it can be differentiated from $R$ to 0 . For ease of reference, this movement is referred to as "embedded object movement".

In order to really understand the nature of the movement in question and distinguish it from $R$ to 0 , we shall choose from the arguments given in support of $R$ to 0 the ones that are felt to be relevant to the discussion of this phenomenon. The first argument we shall be looking at is verb agreement. The most apparent characteristic of embedded object movement is the obligatory presence of the direct object clitic $t$ 'him/her/it' on the embedded verb. This clitic clearly shows that the NP tabrat in (38) is formally related to the embedded verb ara in the sense that tabrat and $t$ are coreferential. This follows from the general principle of Berber which says that whenever a verb is preceded by its lexical direct object NP, i.e. in OVS sentences, a direct object clitic is automatically attached to this verb. Thus, since the moved NP and its corresponding direct object clitic in (38) do not involve any other verb except the CC verb, they must occur within the boundaries of the one and same clause, the CC. The presence of direct object clitics which do not replace lexical direct object NP's but which mark a specific word order formally clearly means that the moved NP belongs to the verb which carries the clitic, and since verbs signal clauses, the fact that tabrat in (38) belongs to ara, and not ssen, seqsa, or iri means that it belongs to the $\mathrm{CC}$ and not to the MC. This constitutes some evidence that no operation across clauses has taken place in (38) and that the sequence tabrat is $t-i$-ara hmad in the examples in question is organised as an independent sentence obeying the principles of ovs sentences in Berber. More evidence comes from the fact that the moved embedded object NP tabrat in (38) cannot be replaced by the direct object clitic in the way the NP raised to the MC direct object position can. Compare (14) and (15) to (39) below:

(39) * $i$-ssen-t muha is $(t)-i$-ara hmad he knew it Muha that it he wrote Ahmed *'Muha knew it that Ahmed wrote (it)' The reason (39) is ill-formed is that in sentences like the ones given under 
(38) for instance, the NP tabrat is not in the MC and therefore is not the MC direct object. Note that (39) is still bad with the NP tabrat in topic position initially:

(40) *tabrat $i$-ssen-t muha is $(t)-i$-ara hmad letter he knew it Muha that it he wrote Ahmed

*'the letter Muha knew it that Ahmed wrote (it)'

Thus, unlike $R$ to 0 which involves the addition of a clitic to the topmost sentence while at the same time not removing it from the embedded sentence, embedded object movement involves the addition of a clitic to the lower CC while at the same time blocking its occurrence in the topmost clause, and since the moved embedded object, unlike the NP raised to object position, cannot be replaced by the direct object clitic, it can hardly be said to belong to it.

The second argument we shall cite is passivisation. Passivisation is a strong and reliable means of differentiating between $R$ to 0 and embedded object movement. The discussion which relates to this topic and which was given in section 3.2 clearly shows that by contrast to a $C C$ subject which is raised to the MC direct object position, a non-subject CC NP (which can only be a direct object since only subjects and objects follow verbs in Berber sentences) cannot passivise. The embedded object is allowed to take part in the process of passivisation only within the boundaries of the CC as stated earlier. Hence, the moved embedded object, unlike the raised NP, is part of the CC and not of the MC. This means that the clause boundary in the three instances given under (38) is not between the NP tabrat and the comp is but between the MC subject muha and the NP tabrat

The third argument that can be invoked in this discussion of the status of the moved embedded direct object NP in relation to $R$ to 0 is reflexivisation. In accordance with what has been said about this rule, namely that it operates when the direct object NP is identical to the subject NP, the moved embedded direct object NP in cases like the ones given under (38) should in principle participate in the rule of reflexivisation since it seems to occupy the direct object position in the MC. However, the following examples show the contrary: 
(41) a. $t$-iyal fadma is i -nya hmad ixf ns she thought Fadma that he killed Ahmed head his

'Fadma thought that Ahmed killed himself'

b. *t -iyal fadma ixf $n s$ is $(t)-i$-nya hmad she thought Fadma head his that it he killed Ahmed

* 'Fadma thought that himself Ahmed killed'

Note that the ungrammaticality of (41b) is accounted for by the fact that this type of sentence is also bad as a simple sentence:

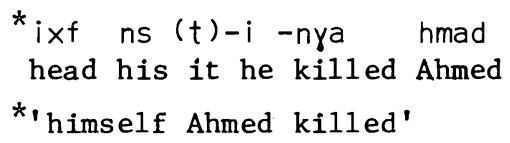

For our purpose, the fact that the moved embedded object NP cannot participate in reflexivising the MC means that it does not belong to it.

A further argument supporting the claim that embedded object movement is different from $R$ to 0 comes from clefting. By way of example, let us cleft the NP tabrat in (38) and see what happens:
tabrat a
i $-\operatorname{ssen}-\left({ }^{*} t\right)$ muha is
* $(t)-i$-ara hmad
letter cleft marker he knew it Muha that it he wrote Ahmed
'it is the letter that Muha knew Ahmed had written'

The obligatory appearance of the direct object clitic on the embedded but not the MC verb is a formal indication that the clefted NP tabrat belongs to the embedded and not to the MC verb.

A final argument which clearly supports the claim that the embedded object movement is different from $R$ to $O$ is that though both types of movement are not recursive, the embedded object movement, unlike $R$ to 0 , can shift the moved NP to the very initial position of a heavily complex sentence. Consider the following possibilities:

(44) a. $\uparrow^{i \text {-bayn }} \uparrow^{t}$-nna fadma ${ }^{i}$-ssen muha is $\uparrow^{i}$-ara hmad tabrat
it seemed she said Fadma he knew Muha that he wrote Ahmed letter
'it seemed that Fadma said that Muha knew that Ahmed wrote the letter' 
b. tabrat $i$-bayn $t$-nna fadma $i$-ssen muha is * $(t)-i$-ara hmad letter it seemed she said Fadma he knew Muha that it he wrote Ahmed *' the letter it seemed that Fadma said that Muha knew that Ahmed wrote'

c. * $i$-bayn tabrat $t$-nna fadma $i$-ssen muha is $(t)-i$-ara hmad it seemed Ietter she said Fadma he knew Muha that it he wrote Ahmed $*^{\prime}$ it seemed the letter Fadma said Muha knew that Ahmed wrote

d. * $i$-bayn $t$-nna fadma tabrat $i$-ssen muha is $(t)$ - $i$-ara hmad it seemed she said Fadma letter he knew Muha that it he wrote Ahmed *'it seemed Fadma said the letter Muha knew that Ahmed wrote'

e. $i$-bayn $t$-nna fadma $i$-ssen muha tabrat is * $(t)-i$-ara hmad it seemed she said Fadma he knew Muha letter that it he wrote Ahmed 'it seemed that Fadma said that Muha knew that Ahmed wrote the letter' Again, the presence of the direct object clitic $t$ in all the well-formed instances is a reminder that the moved embedded object NP is a property of the subordinate $C C$ and not of the MC. Compare this with the case of $R$ to 0 where the embedded subject can move to the initial position providing it leaves a direct object clitic on the main and not the subordinate verb. The boundedness of $R$ to $O$ is also sharply contrasted with the unboundedness of the embedded object movement.

The conclusion that follows from the above discussion of the embedded object movement in relation to $R$ to 0 is that the arguments supporting the latter fail to hold when applied to the embedded object movement. The two movements must, therefore, be different and their difference can be stated in the following terms: whereas $R$ to $O$ is a movement rule which basically involves a change in grammatical relations', i.e. the categorial status of the moved NP, the embedded object movement seems to be nothing more than topicalisation (which will be considered shortly) or some more general re-arranging rule which does not affect grammatical relations, i.e. the categorial status of the moved NP does not change.

4. Raising vs. Topicalisation

Raising in Berber should be differentiated from an apparently similar syntactic operation, topicalisation. First, whereas topicalisation basically in- 
volves movement, Raising, as we saw, involves movement only when the complement subject is raised to the object position of the MC.

Second, whereas topicalisation does not involve any change in the grammatical relations of the moved constituent, Raising involves a change in the grammatical relations of the raised NP, i.e. the former complement subject becomes either subject or object of the MC.

Third, whereas Raising is known to move only NP's, a movement like topicalisation can, in principle, move any other constituent as well.

Fourth, if we push the difference between Raising and topicalisation a 1ittle further, we can say that whereas Raising clearly serves syntactic functions, topicalisation, in not changing the grammatical relations of the moved NP's, serves pragmatic functions more than it serves syntactic functions. In fact, topicalisation involves notions like "topic" and "comment" which are more related to discourse analysis than to sentence analysis. It could even be said that topicalisation does not involve movement. ${ }^{10}$ One could, for example, argue that the NP tabrat in all the instances in which it acts as the moved embedded object starts off under the topic node which is "base-generated". This can be shown as follows:

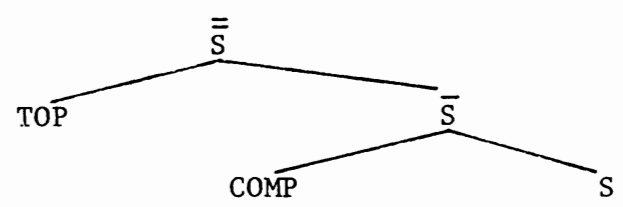

Note incidentally that this type of analysis seems to suit Berber because of the presence of agreement markers in the complement clause, a fact which allows it to occur as an independent sentence following the TOP node.

5. Inadequacy of Chomsky's Framework

Chomsky has argued against $R$ to 0,11 stating that apparent cases of this can be accounted for by more abstract and general principles, i.e. his con-

${ }^{10}$ This is the position which Chomsky maintains. Not all 1 inguists seem to agree with him; for many 1 inguists, movement is involved in topicalisation.

${ }^{11}$ Chomsky [1977] accepts $R$ to $S$ which he subsumes under the general head- 
straints or conditions on rules. ${ }^{12}$ These conditions can be interpreted in either of the following ways: they can be given an absolute interpretation in the sense that what is claimed is that no rule in any language can ever under any circumstance cause violation of any of the constraints which it can be subject to; or these constraints can be given a relative interpretation in the sense that what is claimed is that in a11 languages, all rules are expected to obey the constraints in the neutral or unmarked case though it may happen that in the marked or exceptional case, the formulatian of rules may include the condition that they in fact violate one or more constraints, that is to say, this particular condition has to be built into the formulation of such rules as an inherent part of them. This allows the possibility of having particular rules in given languages "violating" particular constraints.

So far as Berber is concerned, it is the second option which seems to be more appropriate. More specifically, Berber has clear surface violations of at least one of these constraints, the Tensed S Constraint (TSC). This constraint

ing of NP movement/preposing. He allows this rule in non-finite clauses because otherwise it will violate one or more of his general constraints (cf. note 12). For a sentence like 'John seems to be right,' Chomsky proposes the following underlying structure: $\Delta$ seems $[$ John is right ], where $\Delta$ is an empty subject. In constructions which involve the element $i t$, he postulates an underlying it in the same position, though he does not seem to maintain this view in his recent writings.

${ }^{12}$ Following Ross [1967], Chomsky [1977] proposes a set of three constraints which have been subsequently subjected to refinements. For ease of exposition, I sha11 consider the Standard version of these constraints based on Chomsky's well-known article "On WH Movement". These constraints are given below:

1. Subjacency Condition (SC): No constituent can be moved out of more than one containing NP or S node (in any single rule application).

2. Tensed S Condition (TSC): No rule can involve $X$ and $Y$ in structures of

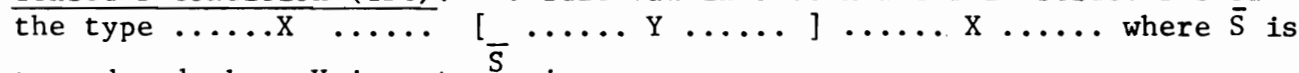
tensed and where $\mathrm{Y}$ is not $\mathrm{S}$ in comp.

3. Specified Subject Constraint (SSC): No rule can involve $X$ and $Y$ in struc-

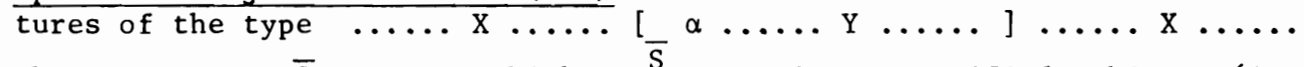
where $\alpha$ is an $\overline{\mathrm{S}}$ or an NP which $\overline{\mathrm{S}}$ contains a specified subject, (i.e. subject not containing $\mathrm{Y}$ and not controlled by $\mathrm{X}$. 
is illustrated by the following example:

(46) a. COMP

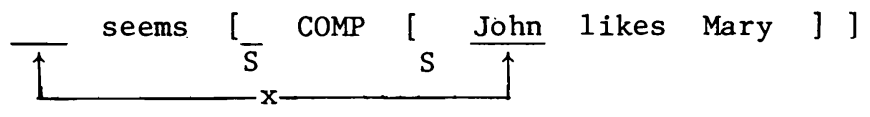

b. * John seems

likes Mary

The NP 'John" in (46a) above cannot be extracted from its clause and be moved to the position marked because this NP originates in a tensed clause, i.e. 'John likes Mary', which is the underlying form, and its movement out of the bracketed tensed $S$ is blocked by virtue of the TSC, hence the ungrammaticality of (46b). By contrast, the movement of the NP 'John' is allowed in structures of the following type:

(47) a. COMP

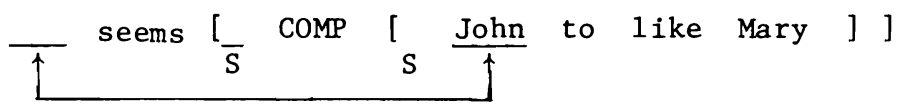

b. John seems to like Mary

The reason is that in (47) above, 'John' is contained within an untensed S. Thus, the extraction of NP's is allowed out of untensed clauses but not out of tensed ones in English.

In Berber, examples 1ike ( $11 b$ ) and (34b) show that extraction of NP's is possible from both finite and non-finite clauses. It might be argued that given the obligatory occurrence of subject agreement markers, $R$ to 0 , 1 ike $R$ to $\mathrm{S}$, is not a chopping but a copying rule and hence is not expected to obey the constraints since the latter operate only in the case of chopping rules. However, the arguments given in support of $R$ to 0 show that this rule involves movement. Further, subject agreement markers in Berber belong to the category $\mathrm{V}$ in the sense that they are an integral part of it. As such, these markers can hardly be seen as filled nodes since on the one hand, they co-occur with their coreferential lexical subject NP's, and on the other hand, having two nodes for the category "subject" is rather redundant. Furthermore, subject agreement markers do not occur in the exact place of subject lexical NP's (they precede, follow, or both precede and follow the verb stem) and hence are not likely to be copies of the lexical subject NP's they co-occur with. 
Berber presents enough evidence for establishing the rule of $R$ to 0 and arguments against Chomsky's views'on this rule can be stated in a sharper way. One might go like this: contrast cases like the following where the embedded subject NP is moved to the matrix object position

(48) i -ssen muha hmad is i -ara tabrat

he knew Muha Ahmed that he wrote letter

'Muha knew that Ahmed had written the letter'

and those where the embedded object NP is moved to the pre-comp position

(49) i -ssen muha tabrat is $t \cdot-i$-ara hmad

he knew Muha letter that it he wrote Ahmed

'Muha knew that Ahmed had'written the letter'

If we assume that transformations (for example Passive) are blind to grammatical relations, 13 then there is nothing to distinguish between hmad in (48) and tabrat in (49). But hmad passivises in the former example and other sentences like it while tabrat in the latter example and other sentences like it cannot as we saw earlier (cf. section 3.2). Why not? There must be some difference between them. The only plausible explanation is that hmad in (48) is in the MC while tabrat in (49) is in the subordinate CC and that grammatical relations are relevant to passive in Berber. If we accept this, then since in a sentence like (48), there is no independent lexical subject NP or subject agreement marker in the embedded clause (the subject agreement marker $i$ of ara being coreferential with hmad in ${ }^{*} i$-ssen muha hmad is $t$-ara tabrat because $t$ here means "she"), hmad must have been raised from that clause. This interpretation of Berber data which assumes the existence of the rule of $R$ to $O$ seems to be more natural and economical than the Chomskyan abstract analysis. The presence of the subject agreement markers and the object clitics renders this view even more so. This claim is also

${ }^{13}$ In most of his works, Chomsky does not use grammatical relations in crucial ways. For him, rules like passivisation and reflexivisation are not inherently restricted to clause internal operations. He uses a system of PRO's, traces (like $e, t$, etc.) to defend his point. 
strengthened by the fact that Chomsky's TSC does not seem to be obeyed in Berber. Even a postulation of traces, PRO's, and the like is far too abstract and removed from the data. Note incidentally that even in so well studied a language as English, not every 1inguist, even from the same theoretical circle, agrees with Chomsky in his approach to $\mathrm{R}$ to 0 .

6. Conclusion

This paper has shown that data from Berber presents interesting facts about the syntactic rule of Raising. On the one hand, Raising to Subject, a rule universally accepted, exists in Berber not as a movement rule but as an agreement copying rule. On the other hand, Raising to Object, a movement rule in Berber, seems to be independently motivated and explains a number of syntactic phenomena in this language, and Chomsky's rejection of this rule does not allow a proper understanding of these syntactic intricacies.

\section{REFERENCES}

Chomsky, N. 1977. "On WH Movement." In P. Culicover, T. Wasow and A. Akmajian (eds.), Formal Syntax, pp. 71-132. New York: Academic Press.

Ennaji, M. and F. Sadiqi. 1986. "The Syntax of Cleft Sentences in Berber." To appear in Studies in Language 10(1).

Ross, J.R. 1967. "Constraints on variables in syntax." Doctoral dissertation, MIT. Available from Indiana University Linguistics Club.

Sadiqi, F. forthcoming. Studies in Berber Syntax. Würzburg: Königshausen \& Newmann. 